\title{
"La escuela confía en que los estudiantes podemos cambiar el mundo": un estudio de las escuelas que trabajan para la justicia social*
}

"The School Trusts That Students Can Change the World": A Study of Schools That Work for Social Justice

"A escola acredita que os estudantes podemos mudar o mundo": um estudo das escolas que trabalham pela justiça social

\author{
Haylen Perines** (ID ORCID ID: 0000-0002-7020-1014 \\ Nina Hidalgo*** (iD ORCID ID: 0000-0002-0056-8566
}

\begin{abstract}
Artículo de investigación
Revista Colombiana de Educación, N. 75. Segundo semestre de 2018, Bogotá, Colombia.

doi: 10.17227/rce.num75-8099

Para citar este artículo: Perines, H. e Hidalgo, N. (2018). "La escuela confía en que los estudiantes podemos cambiar el mundo": un estudio de las escuelas que trabajan para la justicia social. Revista Colombiana de Educación, 75, 19-38.
\end{abstract}

\section{(c) $\underset{\mathrm{BY}}{\mathrm{P}}(\mathrm{NC}$ \\ Recibido: 26/09/2016 \\ Evaluado: 09/10/2017}

* Proyecto financiado por el Ministerio de Economía y Competitividad de España, titulado "Escuelas para la Justicia Social" (EDU2O11-29114), dirigido por F. Javier Murillo de la Universidad Autónoma de Madrid.

** Académica e investigadora del Instituto Interdisciplinario de Ciencia y Tecnología, Universidad de La Serena (Chile). Doctora en Educación de la Universidad Autónoma de Madrid (España). Máster en Gestión Educativa (UMCE, Chile), Profesora de Castellano y Filosofía (ULS, Chile). Correo electrónico: haylen.perines@userena.cl

*** Profesora en la Universidad Autónoma de Madrid. Doctora en Educación en la Universidad Autónoma de Madrid (España). Miembro del grupo de investigación Cambio Educativo para la Justicia Social y de la Red sobre Liderazgo y Mejora Educativa (RILME)

Correo electrónico: nina.hidalgo@uam.es 


\section{Resumen}

Este artículo de investigación expone los resultados de un estudio que buscó identificar las características de las escuelas que trabajan para promover una sociedad más justa desde la perspectiva de los estudiantes. Para hacerlo, se utilizó el grupo de discusión como enfoque metodológico. Se desarrollaron grupos de discusión con estudiantes de cuatro institutos de Educación Secundaria de la Comunidad de Madrid (España), que se constituyen centros altamente heterogéneos en relación con su composición socio-económica y cultural. Los resultados apuntan a que los estudiantes valoran positivamente múltiples aspectos de su escuela, tales como la forma en que se integra a todos sin hacer diferencias, la confianza que los profesores depositan en ellos, la participación de todos los integrantes de la comunidad educativa y la posibilidad que tienen de expresar sus ideas e inquietudes.

\section{Palabras clave}

Justicia Social; Educación: Educación para la Justicia Social; Escuelas para la Justicia Social

\section{Keywords}

Social Justice; Education; Education for Social Justice; Schools for Social Justice
This research article presents the results of a study that sought to identify the characteristics of schools that work to promote a more just society from the perspective of students. To do so, the discussion group was used as a methodological approach. Discussion groups were developed with students from four Secondary Education Institutes of the Community of Madrid (Spain), which are highly heterogeneous centers in relation to their socio-economic and cultural composition. The results indicate that students positively value multiple aspects of their school, such as the way in which they integrate without making differences, the trust that teachers place in them, the participation of all the members of the school community and the possibility that they have to express their ideas

\begin{abstract}
Resumo
Este artigo de pesquisa apresenta os resultados de um estudo que visou identificar as características das escolas que trabalham para promover uma sociedade mais justa desde a perspectiva dos estudantes. Para isso, utilizou-se o grupo de discussão como abordagem metodológica. Desenvolveram-se grupos de discussão com estudantes de quatro instituições de Educação Secundária da Comunidade de Madri (Espanha), que se constituem centros bastante heterogêneos em relação com sua composição socioeconômica e cultura. Os resultados indicam que os estudantes valoram positivamente múltiplos aspectos de sua escola, como a forma na que todos são integrados sem excepções, a confiança que os professores depositam neles, a participação de todos os membros da comunidade educativa e a possibilidade que têm de expressar suas ideias e dúvidas
\end{abstract}




\section{Introducción}

"Si una escuela no se plantea explícitamente la consecución de una sociedad más justa, contribuirá a la reproducción de las injusticias sociales". Con estas palabras, Murillo et al. (2014, p. 3) describen una de las premisas que permiten entender el papel fundamental que tiene la escuela en el camino hacia la justicia social. Pero no es solo una premisa; con sus palabras los autores advierten que si la escuela no genera un cambio interno que conscientemente asuma su responsabilidad como herramienta de cambio, seguirá siendo aquel instrumento que, como han defendido algunos autores, legitima las diferencias sociales y procura mantener a la sociedad dividida en clases (cf. Althusser, 1974; Bourdieu y Paseron, 1972; Plowden, 1969).

Esa escuela que mantiene las desigualdades, ¿es la que queremos para nuestros niños? Desde la mirada de la Justicia Social este interrogante tiene una respuesta evidentemente negativa.

La incidencia de la escuela en la consecución de una sociedad más justa y las características de aquellos centros educativos que trabajan para la justicia social ha despertado el interés de diversos autores (Applebaum, 2004, 2005; Cochran-Smith et al., 2009; Connell, 1997; Enterline, CochranSmith, Ludlow y Mitescu, 2008; Lupton, 2005; Petrou, Angelides y Leigh, 2009; Sensoy y DiAngelo, 2015; Stevenson, 2007), quienes han analizado cómo la escuela intenta compensar las diferencias de partida de los estudiantes, ya sea la situación económica, el género, la raza, la religión, sus capacidades intelectuales, entre otras.

A pesar de este interés por el tema en el ámbito internacional, en España sigue siendo un tópico aún incipiente. No podemos desconocer que existen algunos trabajos teóricos bastante actuales en relación a la justicia social como temática global (cf. Bolívar, 2015; Jacott et al., 2014; Murillo y Hernández-Castilla, 2011a, 2011b, 2014; Murillo e Hidalgo, 2015; Martínez-Garrido y Perines, 2015; Murillo et al., 2014). Sin embargo, no contamos con abundantes investigaciones que aborden esta temática empíricamente, ni tampoco las características de las escuelas que trabajan para la justicia social. Es un desafío pendiente que intentamos abordar en este artículo.

La presente investigación busca identificar las características de las escuelas que trabajan para promover una sociedad más justa desde la perspectiva de los estudiantes de cuatro institutos de educación secundaria de la comunidad de Madrid. 


\section{Marco teórico}

El término justicia social no es fácil de definir, quizás por su carácter político, social y contextual. No se refiere únicamente a derechos humanos, ni a la distribución equitativa de bienes, ni a la igualdad de oportunidades; tampoco existe únicamente dentro del Estado-nación (Murillo y Hernández-Castilla, 2014).

De acuerdo con Fraser (2008), el concepto de justicia social es tridimensional al estar compuesto por: la redistribución, el reconocimiento y la participación (Murillo y Hernández-Castilla, 2011b, 2014):

» La redistribución es entendida como la distribución de los bienes primarios (Rawls, 1971). Esta no se realiza en condiciones de igualdad sino más bien de equidad, es decir, dando más a aquellos que más lo necesitan. En términos sociales, las instituciones deben velar para que se dé este trato diferencial a las personas para compensar las desigualdades creadas por razones socioeconómicas, culturales, de género, capacidad u orientación sexual.

»El reconocimiento se define como el reconocimiento social y cultural de todos los individuos, así como de sus diferentes modos de pensar, hacer y ser (Fraser y Honneth, 2003; Young, 2011). Esta dimensión supone eliminar la dominación cultural existente en la sociedad (donde impera una cultura frente a las otras, tradicionalmente definidas como minoritarias).

» La participación o representación supone velar para que existan las condiciones idóneas para la plena participación de todas las personas en la sociedad, en especial para los grupos que han sido sistemáticamente excluidos por sus características personales y sociales (Fraser, 2008; Young, 2011).

Una de las instituciones que más impacto tiene en la sociedad es la escuela, por lo que si queremos avanzar hacia una sociedad más justa y democrática es necesario empezar por la escuela y por la educación que en ella se imparte. En este sentido, como defienden Murillo y Hernández-Castilla (2011a), es fundamental recuperar el concepto de educación para la justicia social "últimamente sustituido por equidad educativa -mucho más restrictivo y menos ambicioso-, y situarlo en primer término de la agenda política y social" (p. 4).

Murillo y Hernández-Castilla (2014) consideran que es necesario que se den tres premisas al momento de observar la realidad de las escuelas: a) no todas las escuelas son iguales. Mientras que algunas contribuyen al mantenimiento de las desigualdades otras luchan para promover la justicia social, por lo que el papel que desempeñe la escuela es fundamental en la consecución de una sociedad más justa. b) Es necesario que la escuela 
se formule, de forma explícita, luchar contra las desigualdades. c) Las escuelas que luchan para una sociedad más justa se caracterizan por tener una cultura basada en la equidad, democracia y justicia.

En palabras de estos mismos autores, la educación para la justicia social se entiende como:

Una escuela que trabaje para la justicia social debe, a la par, trabajar en justicia social y desde la justicia social. La idea es sencilla, para construir una sociedad más justa es necesario que los alumnos conozcan esos elementos y tengan competencias y capacidades para modificar la situación, pero también mediante una escuela justa. (Murillo y Hernández-Castilla, 2014, pp. 17-18).

Teniendo en cuenta la escuela planteada por Murillo y Hernández-Castilla (2014), consideramos que una escuela para la justicia social se define a partir tres dimensiones complementarias:

» Escuela equitativa, entendida como aquella que garantiza el acceso, la permanencia y el aprendizaje de todos los estudiantes organizando y adaptando los recursos y medios, de tal forma que ayude a aquellos alumnos que más lo necesitan. Asimismo, la educación equitativa evita todas las formas de exclusión, segregación y discriminación en un camino hacia una educación inclusiva, lo que supone una valoración y reconocimiento de las diferencias y fortalezas de los estudiantes (Bolívar, 2005; Darling-Hammond, 2001; Murillo, 2004).

»Escuela democrática, concebida como aquella que es de todos y para todos, y se fundamenta en la libertad social y la igualdad en la diversidad que requiere procesos de deliberativos y de toma de decisión donde la participación y el trabajo cooperativo son esenciales (Apple, 1979; Apple y Beane, 2007; Beane y Apple, 2002; Biesta, 2010; Trafford, 2008).

"Escuela crítica, considerada como la que rompe con las relaciones de clase, las inequidades y la sumisión social y cultural de los individuos. La educación, desde una perspectiva crítica, tiene el poder de transformar y lograr la emancipación social de los sujetos. El propósito de la pedagogía crítica es sensibilizar, denunciar y transformar las situaciones de reproducción, legitimación, dominación y opresión generadas por cuestiones raciales, de clase, género u orientación sexual en el sistema educativo y en la sociedad (Allman, 2001; Giroux, 1990; McLaren, 1984; Kincheloe, 2008). Desde la perspectiva de Smyth (1991, 2001), en una escuela crítica es necesario que: a) los profesores sean intelectuales críticos con el currículum y el proceso de enseñanza; b) los estudiantes sean 
agentes de cambio, y c) la escuela se convierta en una comunidad comprometida con la lucha contra las injusticias.

La investigación internacional ha determinado, por un lado, la incidencia que tiene la escuela en la consecución de una sociedad más justa (Applebaum, 2004, 2005; Cochran-Smith et al., 2009; Connell, 1997; Petrou, Angelides y Leigh, 2009; Sensoy y DiAngelo, 2015; Stevenson, 2007), y, por otro, qué elementos caracterizan a las escuelas que están orientadas a la consecución de la justicia social (Adams y Bell, 2007; Carlisle, Jackson y Alison, 2006; North, 2006), revisamos brevemente cada uno de estos estudios.

Desde la perspectiva de Connie E. North (2006), la justicia social en educación solo puede comprenderse a través de tres binomios claves: igualdad/diferencia, macro/micro y redistribución/reconocimiento. El primero hace referencia a uno de los debates más importantes en torno a una educación socialmente justa, centrado en tratar por igual a todos los estudiantes o bien de forma equitativa, es decir, adaptando la educación a las características y necesidades de cada uno de ellos. Desde la perspectiva de la autora, es fundamental posicionarse en las opciones que individualizan y adaptan la enseñanza, respetando y favoreciendo la diferencia del alumnado. El segundo implica variar la atención en la educación tanto en procesos de nivel macro, como la formulación de políticas educativas y la organización de movimientos sociales como a los procesos a nivel micro, como los comportamientos individuales y las interacciones sociales desarrolladas en el aula. El tercer binomio ahonda en la concepción de la justicia social más cercana a la redistribución de los bienes primarios o bien en el reconocimiento de las particularidades culturales y étnicas de los alumnos. La autora defiende una posición dualista que conciba la educación para la justicia social como aquella que redistribuye los recursos y vela por reconocer y construir la identidad de sus estudiantes.

Complementando el anterior estudio, Adams y Bell (2007) consideran que existen cinco principios para una educación para la justicia social: a) establecer un equilibrio entre los componentes emocionales y cognitivos del proceso de aprendizaje de los estudiantes; b) reconocer y apoyar las características personales e individuales de los alumnos, así como favorecer las interacciones sociales; c) prestar atención explícita a las relaciones sociales dentro del aula; d) favorecer la reflexión y la conciencia crítica de los estudiantes, y e) promover el esfuerzo y el crecimiento personal como base para el desarrollo integral del alumnado.

Uno de los estudios de referencia para nuestra investigación es el de los estadounidenses Carlisle, Jackson y Alison (2006), el cual se enmarca en un proyecto de educación para la justicia social en las escuelas (SJES, por su sigla en inglés) y busca impactar positivamente en la práctica de la 
justicia social en los entornos urbanos. Los autores se proponen identificar cinco principios clave de la educación para la justicia social, a partir del trabajo de una escuela cuyo alumnado está compuesto por estudiantes negros, latinos o pobres. Los cinco principios de que identifican los autores son: inclusión y equidad (aborda todas las formas de opresión social como racismo, sexismo, homofobia, clasismo, etc.), altas expectativas hacia los estudiantes (la escuela proporciona un ambiente que confía en el desarrollo de todos los alumnos), relaciones recíprocas con la comunidad (se busca activamente mantener una relación beneficiosa mutua entre comunidad y familias o agencias locales, en un sistema abierto y accesible) un enfoque de todo el sistema (existe un compromiso de crear un ambiente socialmente justo dentro de todas las áreas del sistema) y compromiso con una educación liberadora (proporciona estrategias para intervenir en situaciones de opresión y busca facilitar el desarrollo de un pensamiento liberador). Estos ayudan a comprender las características de los centros que trabajan para la justicia social.

\section{Metodología}

La presente investigación se enmarca en el proyecto I+D+i "Escuelas para la Justicia Social" (EDU2011-29114) realizado por el grupo de investigación Cambio Educativo para la Justicia Social (GICE), en el periodo 2011-2014. Este trabajo forma parte de uno de los subestudios del proyecto, centrado en la cultura de las escuelas para la justicia social.

El objetivo es identificar las características de las escuelas que trabajan para promover una sociedad más justa desde la perspectiva de los estudiantes. Para lograrlo, realizamos un estudio con el grupo de discusión como enfoque metodológico (Ibáñez, 1992). Buscamos generar y analizar un discurso colectivo a partir de las aportaciones individuales que, en interacción, cobran un nuevo significado.

Se llevaron a cabo cuatro grupos de discusión en cuatro institutos de educación secundaria de la Comunidad de Madrid. Son centros con contextos altamente heterogéneos tanto en composición socioeconómica como cultural de sus estudiantes. Asimismo, son institutos que han sido considerados como centros que trabajan para la justicia social. Para elegir los más adecuados, tuvimos en cuenta aquellas escuelas que eran reconocidas por la comunidad educativa por contar con proyectos de innovación e inclusión educativa, por llevar a cabo iniciativas para terminar con las desigualdades sociales y por tener una especial preocupación por el desarrollo integral de sus estudiantes (atendiendo a sus necesidades emocionales, sociales y académicas). 
Los cuatro grupos de discusión estuvieron compuestos por ocho estudiantes cada uno, que cursaban entre tercero de la educación secundaria obligatoria (ESO) y segundo de bachillerato. Cada uno contaba con chicos y chicas, de diferentes cursos y de distintas procedencias culturales.

Cabe mencionar que los grupos de discusión tuvieron una duración aproximada de una hora y se llevaron a cabo en dependencias de los centros educativos entre los meses de enero y marzo de 2014. Durante su realización fueron grabados bajo el consentimiento de los participantes para posteriormente ser transcritos con la ayuda del programa $F 4$.

Desde la fundamentación teórica hemos establecido algunas categorías de análisis a priori a la entrada al campo, que se complementaron con aquellas que surgían desde los datos recabados. Las categorías iniciales que nos ayudaron a orientar y definir nuestro acercamiento al campo de estudio se basan en las tres dimensiones que definen una escuela para la justicia social: escuela equitativa, escuela democrática y escuela crítica. En ellas incluimos los cinco principios de la educación en justicia social que identifican Carlisle, Jackson y Alison (2006), dado que están en coherencia con las tres dimensiones mencionadas y con el concepto tridimensional de justicia social planteado por Fraser (2008). Los principios de una escuela socialmente justa de Carlisle, Jackson y Alison (2006) puntualizan aspectos y acciones concretos a la luz de las tres dimensiones que proponemos, por lo que utilizarlas en nuestro estudio facilitó el análisis de los grupos de discusión (tabla 1).

Tabla 1. Categorías de análisis

\begin{tabular}{l|l}
\multicolumn{1}{c}{$\begin{array}{c}\text { Dimensiones de una escuela } \\
\text { para la justicia social }\end{array}$} & \multicolumn{1}{c}{$\begin{array}{c}\text { Principios de Carlisle, Jackson } \\
\text { y Alison (2006) }\end{array}$} \\
\hline Escuela equitativa & Inclusión y equidad, \\
Escuela democrática & altas expectativas hacia los estudiantes. \\
Relaciones recíprocas con la comunidad, \\
enfoque de todo el sistema.
\end{tabular}

Fuente: elaboración propia.

Si bien buscábamos que los participantes expresaran sus opiniones sin una estructura particularmente rígida, contábamos con un guion construido previamente para orientar las interacciones. Algunas de las temáticas a abordar se señalan en la tabla 2 . 
Tabla 2. Guion de temas grupos de discusión

Temáticas a abordar
Cambios en la dinámica escolar que han favorecido la calidad del centro.
¿Qué actores han contribuido a generar dichos cambios?
Prácticas de mejoras que se desarrollan en el centro.
La cultura/clima del centro.
Las acciones de mejora que ha desarrollado el equipo directivo en el centro.

Fuente: elaboración propia.

El análisis de la información se basó en la elaboración de sistemas de categorías. Este proceso se inició desde una perspectiva macro que, progresivamente, fue generando categorías más específicas que nos permitieron analizar e interpretar los discursos de nuestros participantes. Después, las categorías se ordenaron jerárquicamente, para identificar las más relevantes. Luego, las unidades, ya jerarquizadas, se agruparon en familias organizadas en códigos que permitieron redactar los resultados. De este modo, se ha seguido un sistema de codificación deductivo e inductivo apoyado con el programa Atlas.ti6, el cual permite tener una visión panorámica de los documentos y trabajar con ellos de manera organizada.

\section{Resultados}

Hemos organizado los resultados del estudio en relación con las tres categorías de análisis que establecimos previamente: escuela equitativa, escuela democrática y escuela crítica, y en coherencia con las categorías descriptivas de la figura 1.

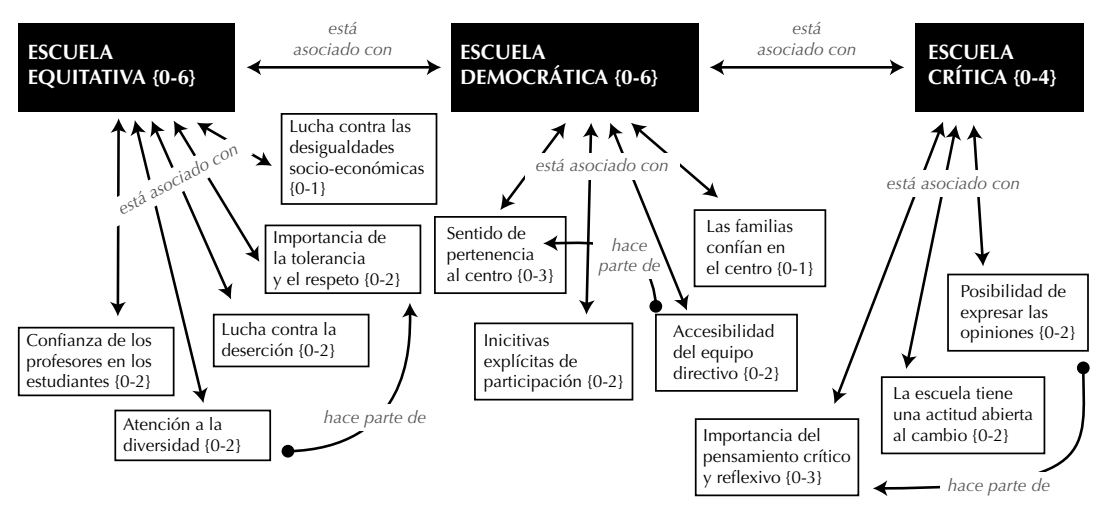

Figura 1. Categorías descriptivas

Fuente: elaboración propia. 


\section{Escuela equitativa}

Los elementos mencionados por los estudiantes pueden ser organizados en dos temas: la atención a la diversidad y la confianza que los profesores depositan en ellos.

Un aspecto que los estudiantes destacan de sus centros educativos es cómo atienden a la diversidad. Las diferencias sociales y personales (como la cultura, el estilo de aprendizaje, la capacidad, el género o la clase social) se hacen imperceptibles porque existen acciones intencionadas por parte de los docentes y directivos para que así sea. Se promueve el trabajo en grupo, no se toleran las discriminaciones, se les explica la importancia de la tolerancia y se llevan a cabo actividades en un clima de armonía donde todas las culturas coexisten de forma empática y solidaria. El instituto trabaja para que todos sean tratados con respeto, dignidad y aprecio, independientemente de sus características particulares:

En este centro siempre intentan que todos convivamos juntos, que nos mezclemos entre sí. Hay gente de muchos lugares y de muchas culturas, pero en realidad no se notan mucho las diferencias, estás acostumbrado a ver a las personas, porque en tu misma clase hay personas rumanas, chinas o de América del Sur, entonces no se ve como algo raro, sino que se ve como algo normal. (C4b:15) ${ }^{1}$.

Para superar las diferencias que puedan generarse en el centro educativo, el equipo directivo y los docentes llevan a cabo distintas iniciativas y actividades. Entre las medidas que mencionan los estudiantes encontramos: a) los desdobles, donde la clase se divide en dos para poder atender de forma más adecuada las necesidades de los estudiantes en grupos más pequeños; b) los alumnos/tutores, quienes ayudan a aquellos estudiantes que tienen más dificultades o que acaban de llegar al colegio; c) el aula de enlace, donde los estudiantes extranjeros aprenden el español:

El año pasado, cuando llegaron dos alumnos chinos, la profesora les ayudaba y les daba otros temas. Les enseñaba lo mismo, pero más fácil para que lo entienda de una manera más sencilla... y también lo hacían con los exámenes. (C1:57).

Aquí tenemos un aula de enlaces donde se les enseña español a todos aquellos que nunca han hablado el idioma y después se integran a nuestra aula. También si te cuesta un poco tienes la opción del "desdoble", donde la profesora trabaja con pocos alumnos donde aprendes más. (C3:47).

1 De este modo se señala la línea de la entrevista, derivada de la transcripción y análisis en el software Atlas.ti. La letra C es la abreviación de la palabra centro. 
Los estudiantes valoran las medidas específicas que toma la escuela para luchar contra las desigualdades socioeconómicas. El centro apoya y ayuda a quienes tienen más problemas económicos, sociales y familiares a través de iniciativas como los libros socializados, la participación de los estudiantes con asociaciones del barrio o el voluntariado. Por ejemplo, el centro financia la excursión a los estudiantes que no pueden hacer frente a ese gasto, como nos comenta un estudiante:

Por ejemplo, si tenemos que ir de excursión y un alumno no tiene dinero, el centro le da ese dinero para que pueda ir. Y eso nadie lo desconoce y a nadie le asombre, es algo habitual para nosotros. (C1:95).

Con una iniciativa similar, uno de las escuelas ha creado un banco de alimentos para recolectar comida y dársela a aquellas familias del centro que presentan más dificultades económicas. Los estudiantes aprecian este tipo de iniciativas, ya que consideran que la escuela puede aportar en la mejora de la vida de sus compañeros y de su entorno:

Desde el departamento de orientación ayudan a las familias que no tienen recursos, que tienen más dificultades. Por ejemplo, este año, si tu familia está pasando problemas de dinero hacemos un banco de alimentos que se va entregando a quienes lo necesitan. (C2:28).

Asimismo, la escuela se preocupa por que los estudiantes no dejen los estudios. Las características de los estudiantes, su situación familiar o económica hace que, en ciertos casos, algunos de ellos quieran abandonar la escuela. Desde la dirección se trabaja para impedir que abandonen la escuela y continúen con su formación. En palabras de una estudiante:

Siempre intentan que no lo dejes que no te quedes atrás, sino que sigas. [...] Por ejemplo, el año pasado vino una chica a darnos una charla sobre la motivación en el estudio y era gitana, y nos contó su experiencia, cómo tuvo que contar en su casa de que quería seguir estudiando. Esta chica ha llegado lejos. A mi amiga, por ejemplo, le pusieron muchas pegas porque se incorporó tarde y ya habían empezado las clases, pero desde dirección y el departamento de orientación le dijeron que le iban ayudar, que no se preocupase. Por eso digo que a todos nos echan un cable, nos animan, nos empujan a que sigamos adelante. (C2:201).

Otro de los elementos que mencionan los estudiantes es la confianza que demuestran los profesores en cada uno de ellos, lo que se traduce no solo en el ámbito académico sino también en el personal. Los docentes constantemente transmiten que confían en sus capacidades y que creen en ellos como personas, sintiéndose aceptados con sus potencialidades y con sus debilidades. Esto genera una confianza recíproca y una relación positiva que favorece un buen clima de trabajo en el centro. Las palabras de algunos estudiantes son las siguientes: 
Los profesores no solo se interesan en lo que tenemos que aprender, sino que también en nosotros como personas. Siempre nos animan a creer en nosotros, aunque pensemos que no podemos hacer las cosas. (C2:88)

Los profesores dicen: "Tienes que estudiar más porque yo sé que tú puedes yo sé que tú eres listo, y si te esforzaras sacarías muy buena nota". Los profesores te apoyan, te refuerzan bastante y hacen bien en decirlo. Por ejemplo, yo en $1^{\circ}$ de la ESO no hacía nada de nada [...] una profesora en el recreo me dijo que quería hablar conmigo y lo que me dijo fue genial, me dijo que confiaba en mí, que yo podía lograrlo. (C2:89).

La confianza de los profesores les hace sentir que su centro es diferente a otros institutos:

Pues yo, a veces, cuando hablo, pues no sé, me siento como orgullosa porque viendo otros institutos comparo el mío y obviamente, me gusta más el mío claro, pero no sé, por todo lo que han dicho mis compañeros... que los profesores están pendientes de ti. (C1:79).

Los estudiantes también aprecian el apoyo constante que les dan los docentes al mostrarse interesados en lo que piensan y sienten, por lo que se sienten apoyados y escuchados:

La persona que somos es lo más importante, nos lo dicen en todas las clases. Acá los profesores se preocupan de nosotros como personas. Son como un apoyo más, prácticamente pasamos más tiempo aquí que en nuestra casa y es un gran apoyo saber que puedes contar con ellos cuando tengas algún problema. (C3:17).

Los adolescentes constantemente reciben consejos y palabras de aliento de parte de sus profesores, quienes intentan orientarlos en las decisiones que deben tomar. Un ejemplo de los consejos que reciben es expresado por estudiantes de cuarto año de la ESO quienes están en una compleja etapa de decisiones en relación a su futuro, por lo que valoran cuando los profesores les hacen reflexionar sobre las opciones que tienen para decidir. Los docentes los motivan a que persigan sus sueños y metas, esforzándose y dando lo mejor de sí:

Yo, por ejemplo, que vengo de otro instituto, creo que en este instituto hay mucha más implicación. En el anterior, yo me acuerdo que había profesores que sí se preocupaban, pero había otros que te decían: "Búscate la vida, tú sabrás". Aquí, sin embargo: "Piénsatelo, inténtalo, hazlo, lo ves lo pruebas, y si no te gusta pues ya te buscas por otra cosa". (C4B:74).

Los estudiantes valoran que en el centro educativo se lleven a cabo proyectos, actividades e iniciativas que buscan el desarrollo no solo de los aprendizajes tradicionales, sino de ellos como personas, para que salgan al mundo siendo ciudadanos que saben pensar: 
Recuerdo la charla que nos dieron al llegar al instituto para empezar la ESO. La jefa de estudios dijo que da igual que salgáis de aquí sabiendo de números o letras. Lo más importante es que salgamos de aquí siendo personas, que fuésemos parte de la sociedad, lo que abarca también saber pensar, saber razonar. (C2:149).

La forma innovadora en que los profesores dan sus clases también es un aspecto destacado por los estudiantes. Sus docentes les plantean actividades creativas y desafiantes buscando que todos aprendan, respetando los diferentes ritmos de aprendizaje o las características particulares de cada uno. Les agrada que sus profesores hagan esfuerzos para que todos puedan acceder a los conocimientos por medio de metodologías menos tradicionales:

Por ejemplo, ha llegado un nativo de Florida, entonces con él hacemos juegos, en la clase de lengua hacemos murales para aprender, también hacemos exposiciones en historia y otras cosas diferentes para no estar sentados escribiendo todo el tiempo, porque no todos aprendemos así. (C3:118).

\section{Escuela democrática}

Uno de los aspectos destacados por los estudiantes que hacen de la escuela un espacio democrático es la implicación de los miembros del equipo directivo en la escuela. Los adolescentes señalan que la escuela es de todos y por ello se implican en su funcionamiento y gestión.

Esa implicación se traduce en la participación de todos los integrantes de la comunidad educativa. Para promover dicha participación, el centro desarrolla diversas iniciativas y programas. Son escuelas que planifican diversas dinámicas, donde hay espacios importantes para charlas, concursos, seminarios o talleres:

El centro hace muchas actividades donde los alumnos podemos participar. Estamos implicados en proyectos a los que te pueden apuntar, y tienen en cuenta todo lo que dices. Por ejemplo, la revista digital, los concursos literarios, los concursos de matemáticas, concursos de arte... intentan que cada alumno destaque un poco y que todos tengan la posibilidad de participar. (C2:36).

La participación de la comunidad también incluye a las familias. Los estudiantes mencionan que los profesores invitan a los padres y madres a participar en el centro, lo que es bien recibido por las familias, las cuales confían en el trabajo del instituto y se sienten conformes con la educación que están recibiendo sus hijos: 
Que te vea tu padre o tu madre llegar contento del instituto, pues eso hace mucho. Que vean que tienes buena relación con los compañeros, con los profesores, que, si necesitas algo, vas a estar, sabes, están seguros de que aquí estás seguro. Esas horas que estás aquí, están tranquilos. (C4b:110).

Los estudiantes valoran que los espacios físicos del instituto estén disponibles para todos, incluso fuera del horario de clases. Ocupar las instalaciones del centro les hace creer que pertenecen a él, sentirse importantes y valorados. Además, algunas de estas escuelas están abiertas a la comunidad en caso de que alguna asociación, grupo de padres o vecinos del barrio necesiten un espacio para llevar a cabo sus actividades, lo que también es visto como algo positivo por los estudiantes:

Si necesitas algo te lo dan. Por ejemplo, si para hacer algún trabajo necesitamos el centro por la tarde nos dejan venir, nos dejan los gimnasios, las salas, las aulas... Y eso está muy bien. Si necesitas el centro para hacer cualquier tipo de actividad no hay ningún problema, incluso si no eres alumno. Si eres un vecino que necesita un espacio para una reunión o algo similar el centro facilita los espacios. (C2:117).

Otro de los aspectos señalados por los estudiantes, es la accesibilidad del equipo directivo. La dirección tiene siempre la puerta abierta para que docentes, estudiantes y familias puedan hablar con ellos o exponerles cualquier situación. Esta accesibilidad es también personal, por lo que el director, jefe de estudios y secretario escuchan a los estudiantes y se preocupan por buscar de forma conjunta una solución a sus problemas:

Los miembros del equipo directivo son muy cercanos. Si quieres hablar con ellos o necesitas algo puedes entrar a su despacho. Por el pasillo muchas veces hablo con la directora, con la jefa de estudios, además me han dado clase, las conozco desde hace varios años. (C2:64).

Por último, los adolescentes señalan que tanto ellos como sus familias pueden comunicarse de forma abierta y sencilla con el centro educativo. Esta comunicación es fluida y permite que las familias y los estudiantes participen en la escuela. Para ello, el equipo directivo pone a disposición de las familias distintos canales de comunicación como el teléfono, el correo electrónico, la página web o bien ir al despacho del director a tratar cualquier tema que necesiten:

El teléfono, la página web, la revista digital, el mural de la puerta, la plataforma de la comunidad. En el mural podemos escribir libremente todos lo que queramos. Todo lo que sucede es hablado entre nuestros padres y el tutor. Además, los padres vienen a las reuniones generales, lo demás se habla por mail o por teléfono. (C2:21) 
Cuando hay algún problema, el centro llama rápidamente a los padres para contárselo, para que vengan aquí al centro. Cuando llegas también hay tutorías, pero si el alumno va bien, a lo mejor una por curso y ya está. (C3:150).

\section{Escuela crítica}

Los estudiantes piensan que en sus centros tienen la posibilidad de expresar sus ideas, ya que pueden opinar sobre lo que piensan o lo que quieren sin sentirse avergonzados. Para ellos, su instituto es un espacio abierto donde tienen libertad para manifestar sus emociones o inquietudes bajo los acuerdos de convivencia y respeto previamente establecidos:

Antes, había círculos de convivencia, buenos los sigue habiendo. Entonces en los círculos de convivencia hay personas que representan a cada clase. Se habla de los problemas que hay entre compañeros, si hay algún conflicto que resolver. Luego los representantes se reúnen con el orientador o con las personas que lo organicen, los comentan e intentan mejorar la situación. (C4a:219).

La escuela trabaja para que los estudiantes piensen y reflexionen, tanto sobre su proceso de aprendizaje como de todo lo que les rodea. Así, los estudiantes indican que el centro, además de escuchar sus ideas e incluirlas, también promueve que sean críticos con el entorno, especialmente con aquellas situaciones injustas o que generan desigualdad. Los estudiantes sienten que el centro trabaja para que tengan una actitud abierta al cambio.

Los adolescentes sienten que el centro favorece que se impliquen con la comunidad mediante una participación activa en distintas asociaciones. Estas entidades tienen una clara orientación social y se centran en la ayuda a personas enfermas, a personas mayores, a niños sin hogar o cuidado de animales:

Hay algunos voluntariados muy bonitos. Por ejemplo, hay uno asociado a la protección animal, también está ese de los niños más pequeños que necesitan reforzar las materias, hay otro donde se trabaja en hospitales, residencias de abuelos, también hay otro que se llama el banco de tiempo. (C4a:89).

En definitiva, los estudiantes consideran que la escuela no es un lugar donde únicamente van a adquirir aprendizajes instrumentales, sino en el que se desarrollan como personas y aprenden a ser responsables de ellos mismos y del mundo que les rodea. 


\section{Discusión}

En términos generales, los hallazgos del presente estudio respaldan el concepto tridimensional de justicia social construido a partir de las dimensiones de redistribución, reconocimiento y participación (Fraser, 2008, Murillo y Hernández-Castilla, 2014).

En coherencia con la redistribución de los bienes primarios (Rawls, 1971), los estudiantes indican que en sus escuelas se ayuda a quienes más lo necesitan, no solo en lo socioeconómico, sino en aspectos culturales, de género o de capacidad. Son instituciones que intentan dar un trato diferencial a las personas para compensar las desigualdades. Asimismo, los alumnos se sienten valorados y respetados con sus particularidades y diferencias, lo que se relaciona con el reconocimiento social y cultural de todos los individuos, y de sus diferentes modos de ser y de pensar (Fraser y Honneth, 2003; Young, 2011). En cuanto a la participación o representación, los estudiantes destacan las oportunidades que les da el centro para que toda la comunidad pueda participar tanto en la escuela como en la sociedad (Fraser, 2008; Young, 2011).

Los resultados de este estudio son coherentes con los tres binomios propuestos por North (2006) para comprender la justicia social en educación: trato equitativo a todo el alumnado, los movimientos e interacciones sociales y el reconocimiento a sus particularidades. También encontramos puntos de encuentro entre lo indicado por los estudiantes de este trabajo y los cinco principios básicos de Adams y Bell (2007), especialmente a la importancia de reconocer las características individuales y al fomento del desarrollo integral del alumnado.

Hemos indicado que el estudio de Carlisle, Jackson y Alison (2006) es un trabajo de referencia en la fundamentación teórica y metodológica de esta investigación. La vinculación de nuestros hallazgos con los cinco principios de la educación para la justicia social, expuestos en dicho estudio es evidente. Los alumnos que participaron en esta investigación destacaron aspectos como la integración de todos por igual (inclusión y equidad), la confianza que tiene el centro en lo que puedan lograr (altas expectativas hacia los estudiantes), la buena comunicación que existe con las familias (relaciones recíprocas con la comunidad), la participación de todos en una variedad de actividades (un enfoque de todo el sistema) y la importancia de que desarrollen un pensamiento crítico y autónomo (compromiso con una educación liberadora). 


\section{Conclusiones}

Los hallazgos de este estudio son un interesante aporte empírico respecto a aquellos elementos que caracterizan a las escuelas que trabajan para la justicia social. Las altas expectativas hacia los estudiantes, la participación de la comunidad o la inclusión equitativa de todos los alumnos dan indicios de que es posible construir escuelas que se hagan conscientes de las injusticias y que intenten superarlas por medio de una educación comprometida con la sociedad.

Centrar nuestra mirada en la voz de los estudiantes implica analizar las escuelas desde la perspectiva de quienes son los claros protagonistas del proceso de aprendizaje, lo que enriquece nuestro análisis. Escuchar la voz de los estudiantes supone observar la realidad educativa desde las opiniones e inquietudes de quienes día tras día merecen una educación socialmente justa.

Las posibles líneas de investigación que pueden surgir a partir de este estudio son, por un lado, llevar a cabo un estudio cuantitativo ex post facto con el objetivo de identificar las características de una escuela que lucha contra las injusticias sociales, y por otro, realizar estudios complementarios de corte cualitativo para triangular la presente investigación con la visión que aporte el resto de miembros de la comunidad educativa: equipo directivo, docentes y familias.

Seguir profundizando en cómo tienen que ser los centros que trabajan para la justicia social es una tarea urgente para terminar con las inequidades que nos rodean. Luchar contra las injusticias no está únicamente en manos de los gobiernos, los responsables políticos y las administraciones, sino que la escuela tiene mucho qué decir y hacer. En palabras de Murillo y Hernández-Castilla (2014):

Los que trabajamos en el mundo educativo tenemos una responsabilidad ética de luchar por un mundo más justo. Es lo que da sentido a nuestro trabajo. Cómo nos organicemos, qué enseñemos, y cómo nos relacionamos con los colegas y con nuestros estudiantes, qué soñamos y qué hacemos para conseguirlo [...] todo ello contribuirá a la conformación de una sociedad u otra. En nuestras manos está colaborar para lograr una mayor justicia social o permitir, por acción o negligencia, el desarrollo de las injusticias. (p. 29). 


\section{Referencias}

Adams, M. y Bell, L.A. (2007). Pedagogical framework for social justice education. En M. Adams, L.A. Bell y P. Griffin (eds.), Teaching for diversity and social justice (pp. 15-34). Nueva York: Routledge.

Allman, P. (2001). Critical education against global capitalism: Karl Marx and revolutionary critical education. Westport, CT: Bergin \& Garvey.

Althusser L. (1974). Ideología y aparatos ideológicos de Estado (Notas para una investigación). En L. Althusser (ed.), Escritos (pp. 105-170). Barcelona: Laia.

Apple, MW. (1979). Ideology and curriculum. Boston: Routledge and Kegan \& Paul.

Apple, M. y Beane, J.A. (2007). Lessons in democratic schools. En M. Apple y J.A. Beane (eds.), Democratic schools: Lessons in powerful education (pp. 150-155). Portsmouth, NH: Heinemann.

Applebaum, B. (2004). Social justice education, moral agency, and the subject of resistance. Educational Theory, 54(1), 59-72.

Applebaum, B. (2005). In the name of morality: Moral responsibility, whiteness and social justice education. Journal of Moral Education, 34(3), 277-290.

Beane, M. y Apple, M. (2002). Escuelas democráticas. Madrid: Morata.

Biesta, G.J. (2010). How to exist politically and learn from it: Hannah Arendt and the problem of democratic education. Teachers College Record, 112(2), 556-575.

Bolívar, A. (2005). Equidad educativa y teorías de la justicia. REICE: Revista Electrónica Iberoamericana sobre Calidad, Eficacia y Cambio en Educación, 3(2), 42-69.

Bolívar, A. (2015). Justicia social y equidad escolar. Una revisión actual. Revista Internacional de Educación para la Justicia Social (RIEIS), 1(1),9-45.

Bourdieu, P. y Passeron, J. (1972). La reproducción: elementos para una teoría del sistema de enseñanza. Barcelona: Laia.

Carlisle, L., Jackson, B. y Alison, G. (2006). Principles of social justice education: the social justice education in schools project. Equity \& Excellence in Education, 39(1), 55-64.

Cochran-Smith, M., Shakman, K., Jong, C., Terrell, D.G., Barnatt, J. y McQuillan, P. (2009). Good and just teaching: The case for social justice in teacher education. American Journal of Education, 115(3), 347-377.

Connell, R.W. (1997). Escuelas y justicia social. Madrid: Morata.

Darling-Hammond, L. (2001). El derecho de aprender. Crear buenas escuelas para todos. Barcelona: Ariel. 
Enterline, S., Cochran-Smith, M., Ludlow, L.H. y Mitescu, E. (2008). Learning to teach for social justice: Measuring change in the beliefs of teacher candidates. The New Educator, 4(4), 267-290.

Fraser, N. (2008). La justicia social en la era de la política de identidad: redistribución, reconocimiento y participación. Revista de Trabajo, 4(6), 83-99.

Fraser, N. y Honneth, A. (2003). ¿Redistribución o reconocimiento?: Un debate político filosófico. Madrid: Morata.

Giroux, H. (1990). La educación social en el aula: la dinámica del currículum oculto en los profesores como intelectuales: Hacía una pedagogía crítica del aprendizaje. Barcelona: Paidós.

Ibáñez, J. (1992). Más allá de la sociología: el grupo de discusión, técnica y crítica. Madrid: Siglo xxı.

Jacott, L. et al. (junio de 2014). Representations of Social Justice of Spanish teachers and students. Comunicación presentada en Sixteenth Annual CiCe Network Conference. Olsztyn: Polonia.

Kincheloe, J.L. (2008). La pedagogía crítica en el siglo xxı: Evolucionar para sobrevivir. En P. McLaren y J.L Kincheloe (eds.), Pedagogía crítica. De qué hablamos, dónde estamos (pp. 25-69). Barcelona: Graó.

Lupton, R. (2005). Social justice and school improvement: improving the quality of schooling in the poorest neighbourhoods. British Educational Research Journal, 31(5), 589-604.

McLaren, P. (1984). Una introducción a la pedagogía crítica en los fundamentos de la educación. México: Siglo xxı.

Martinez-Garrido, C. y Perines, H. (junio de 2015). Estudio de las actitudes de los futuros docentes sobre justicia social. Comunicación presentada en XVII Congreso Internacional de Investigación Educativa: Investigar con y para la sociedad. Cádiz: España.

Murillo, F.J. (2004). Equidad en educación. REICE. Revista Iberoamericana sobre Calidad, Eficacia y Cambio en Educación 2(1), 1-4.

Murillo, F.J. y Hernández-Castilla, R. (2011a). Trabajar por la justicia social desde la educación. REICE. Revista Electrónica Iberoamericana sobre Calidad, Eficacia y Cambio en Educación, 9(4), 3-6.

Murillo, F.J. y Hernández-Castilla, R. (2011b). Hacia un concepto de justicia social. REICE. Revista Iberoamericana sobre Calidad, Eficacia y Cambio en Educación, 9(4), 7-23.

Murillo, F.J. y Hernández-Castilla, R. (2014). Liderando escuelas justas para la justicia social. Revista Internacional de Educación para la Justicia Social (RIEIS), 3(2), 13-32.

Murillo, F.J. e Hidalgo, N. (2014). Enfoques fundamentales de la evaluación de estudiantes para la justicia social. Murillo, F.J., Hernández-Castilla, R., Hidalgo, N., Paez-Lacadena, P., Cerrillo, R. y Tello, 
I. School Culture, key for Social Justice Schools. Comunicación presentada en el Congreso ECER 2014. The Past, Present and Future of Educational Research in Europe. Porto: Portugal.

Murillo, F. J. e Hidalgo, N. (2015). Enfoques Fundamentales de la Evaluación de Estudiantes para la Justicia Social. Revista Iberoamericana de Evaluación Educativa, 8(1), 43-61.

North, C.E. (2006). More than words? Delving into the substantive meaning (s) of "social justice" in education. Review of Educational Research, 76(4), 507-535.

Petrou, A., Angelides, P. y Leigh, J. (2009). Beyond the difference: From the margins to inclusion. International Journal of Inclusive Education, 13(5), 439-448.

Plowden, B. (1969). Children and Their Primary Schools: A Report. Research and Surveys: HM Stationery Office.

Rawls, J.A. (1971). A Theory of Justice. Cambridge, MA: Harvard University Press.

Sensoy, Ö. y DiAngelo, R. (2015). Is everyone really equal?: An introduction to key concepts in social justice education. Nueva York: Teachers College Press.

Smyth, J. (2011). Critical pedagogy for Social Justice. Londres: Continuum.

Smyth, J. (1991). Una pedagogía crítica de la práctica en el aula. Revista de Educación, 294, 275-300.

Stevenson, H.P. (2007). A case study in leading schools for social justice: when morals and markets collide. Journal of Educational Administration, 45(6), 769-781.

Trafford, B. (2008). Democratic Schools. En J. Arthur, I. Davies y C. Hahn (eds.), SAGE Handbook of Education for Citizenship and Democracy (pp. 410-423). Londres: Sage Publishing.

Young, I.M. (2011). Responsabilidad por la justicia. Madrid: Paidós. 